\title{
Mechanical Thrombectomy for Isolated M2 Occlusions: A Post Hoc Analysis of the STAR, SWIFT, and SWIFT PRIME Studies
}

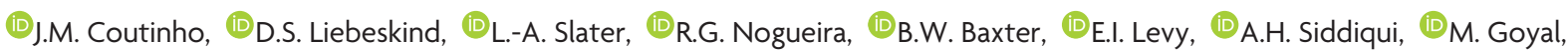

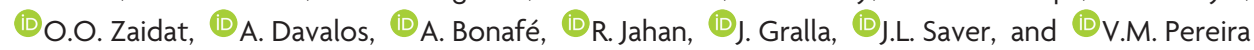

\begin{abstract}
BACKGROUND AND PURPOSE: Mechanical thrombectomy is beneficial for patients with acute ischemic stroke and a proximal anterior occlusion, but it is unclear if these results can be extrapolated to patients with an M2 occlusion. The purpose of this study was to examine the technical aspects, safety, and outcomes of mechanical thrombectomy with a stent retriever in patients with an isolated M2 occlusion who were included in 3 large multicenter prospective studies.
\end{abstract}

MATERIALS AND METHODS: We included patients from the Solitaire Flow Restoration Thrombectomy for Acute Revascularization (STAR), Solitaire With the Intention For Thrombectomy (SWIFT), and Solitaire With the Intention for Thrombectomy as Primary Endovascular Treatment (SWIFT PRIME) studies, 3 large multicenter prospective studies on thrombectomy for ischemic stroke. We compared outcomes and technical details of patients with an $\mathrm{M} 2$ with those with an $\mathrm{Ml}$ occlusion. All patients were treated with a stent retriever. Imaging data and outcomes were scored by an independent core laboratory. Successful reperfusion was defined as modified Thrombolysis in Cerebral Infarction score of $2 b / 3$.

RESULTS: We included 50 patients with an M2 and 249 patients with an M1 occlusion. Patients with an M2 occlusion were older (mean age, 71 versus 67 years; $P=.04$ ) and had a lower NIHSS score (median, 13 versus 17; $P<.001$ ) compared with those with an M1 occlusion. Procedural time was nonsignificantly shorter in patients with an $\mathrm{M} 2$ occlusion (median, 29 versus 35 minutes; $P=.41$ ). The average number of passes with a stent retriever was also nonsignificantly lower in patients with an $\mathrm{M} 2$ occlusion (mean, 1.4 versus $1.7 ; P=.07$ ). There were no significant differences in successful reperfusion ( $85 \%$ versus $82 \%, P=.82$ ), symptomatic intracerebral hemorrhages ( $2 \%$ versus $2 \%, P=1.0$ ), device-related serious adverse events (6\% versus $4 \%, P=.46)$, or modified Rankin Scale score $0-2$ at follow-up (60\% versus $56 \%, P=.64)$.

CONCLUSIONS: Endovascular reperfusion therapy appears to be feasible in selected patients with ischemic stroke and an M2 occlusion.

ABBREVIATIONS: ESCAPE = Endovascular Treatment for Small Core and Proximal Occlusion Ischemic Stroke; IMS = Interventional Management of Stroke; $\mathrm{MERCI}=$ Mechanical Embolus Removal in Cerebral Ischemia; MT = mechanical thrombectomy; REVASCAT = Endovascular Revascularization With Solitaire Device Versus Best Medical Therapy in Anterior Circulation Stroke Within 8 Hours; STAR = Solitaire FR Thrombectomy for Acute Revascularization; SWIFT = Solitaire FR With the Intention For Thrombectomy; SWIFT PRIME = Solitaire With the Intention for Thrombectomy as Primary Endovascular Treatment

$\mathbf{R}$ ecent data have shown that mechanical thrombectomy (MT) with a stent retriever is safe and improves functional outcome in patients with acute ischemic stroke and an occlusion of the anterior circulation. ${ }^{1-5}$ It is unclear, however, if these results can

\section{Received July 1, 2015; accepted August 11}

From the Divisions of Neuroradiology (I.M.C., L.-A.S., V.M.P.) and Neurosurgery (V.M.P.), Department of Medical Imaging and Department of Surgery, Toronto Western Hospital, University Health Network, University of Toronto, Ontario, Canada; Neurovascular Imaging Research Core and the University of California, Los Angeles Stroke Center (D.S.L.), Los Angeles, California; Marcus Stroke and Neuroscience Center (R.G.N.), Department of Neurology, Grady Memorial Hospital, Emory University School of Medicine, Atlanta, Georgia; Department of Radiology (B.W.B.), Erlanger Hospital at University of Tennessee, Chattanooga, Tennessee; Department of Neurosurgery (E.I.L., A.H.S.) and Toshiba Stroke and Vascular Research Center (A.H.S.), State University of New York at Buffalo, Buffalo, New York: Departments of Radiology and Clinical Neurosciences (M.G.), University of Calgary, Calgary, Alberta, Canada; Department of Neurology (O.O.Z.), Medical College of Wisconsin, Milwaukee, Wisconsin; Department of Neurosciences (A.D.), Hospital Germans Trias i Pujol, Universitat Autònoma de Barcelona, Badalona, Barcelona, Spain; Department of Neuroradiology (A.B.), Hôpital Gui-de-Chauliac, Montpellier, be extrapolated to patients with an occlusion of the second segment of the middle cerebral artery (M2 occlusion). Because of its distal location, smaller diameter, and thinner walls, MT of the M2 segment is technically more challenging and may be associated with a higher risk of periprocedural complications. The potential benefit of reperfusion may also be different, in part because M2 occlusions generally respond better to IV thrombolysis. ${ }^{6}$

France; Division of Interventional Neuroradiology (R.J.) and Department of Neurology and Comprehensive Stroke Center (J.L.S.), David Geffen School of Medicine, University of California, Los Angeles, Los Angeles, California; and Departments of Diagnostic and Interventional Neuroradiology (J.G.), Inselspital, Bern University Hospital and University of Bern, Bern, Switzerland.

This work was supported by the STAR and SWIFT studies, funded by Covidien.

Paper previously presented at: European Stroke Organization Congress, April 17-19, 2015; Glasgow, UK.

Please address correspondence to V.M. Pereira, MD, Room 3MCL-436, Toronto Western Hospital, 399 Bathurst St, Toronto, ON, M5T 2S8, Canada; e-mail: vitormpbr@hotmail.com

http://dx.doi.org/10.3174/ajnr.A4591 


\begin{tabular}{cc} 
MCA Segment & Anatomic Definition \\
\hline M1 & $\begin{array}{l}\text { Horizontal segment of the proximal MCA from the bifurcation of the ICA into the anterior and middle cerebral arteries to } \\
\text { the genu of the MCA branch or branches at the entrance to the insula } \\
\text { Vertical MCA branches in the Sylvian fissure originating at the genu and extending to the next genu at the level of the } \\
\text { operculum; if the anterior temporal artery arises from the horizontal M1 segment, it will not be } \\
\text { considered an M2 branch }\end{array}$ \\
Branches that continue when the M2 branches change course again to a more lateral/horizontal course in the \\
Sylvian fissure away from the insula and below the operculum \\
Branch vessels primarily extend vertically over the frontal and parietal lobes and inferiorly over the temporal lobe
\end{tabular}

The number of patients with an M2 occlusion in the thrombectomy trials was small. The Multicenter Randomized Clinical trial of Endovascular Treatment for Acute Ischemic Stroke in the Netherlands (MR CLEAN) protocol did allow recruitment of these patients, but $<8 \%$ of included patients had an isolated M2 occlusion. ${ }^{1}$ In the Endovascular Revascularization With Solitaire Device Versus Best Medical Therapy in Anterior Circulation Stroke Within 8 Hours (REVASCAT), Endovascular Treatment for Small Core Proximal Occlusion Ischemic Stroke (ESCAPE), and Extending the Time for Thrombolysis in Emergency Neurological Deficits-Intra-Arterial (EXTEND-IA), only 10, 6, and 4 patients with an isolated M2 occlusion were treated with MT, respectively. ${ }^{2,4,5}$ The Solitaire With the Intention for Thrombectomy as Primary Endovascular Treatment (SWIFT PRIME) protocol excluded M2 occlusions, but there were some protocol violations. ${ }^{3}$ Thus far, none of these studies has specifically examined the subgroup of M2 occlusions. The aim of our study was to examine the technical aspects, safety, and outcomes of MT with a stent retriever in patients with an isolated M2 occlusion who were included in 3 large multicenter prospective studies.

\section{MATERIALS AND METHODS \\ Description of Studies and Patient Selection}

We included patients from the Solitaire FR With the Intention for Thrombectomy (SWIFT) trial, Solitaire FR Thrombectomy for Acute Revascularization (STAR) study, and the SWIFT PRIME trial. The design of these studies has been reported previously. ${ }^{3,7-9}$ Briefly, the SWIFT trial was a randomized clinical trial that ran from 2010 to 2011, in which patients with acute ischemic stroke and an angiographically confirmed occlusion of a the proximal cerebral artery were allocated to treatment with a stent retriever (Solitaire; Covidien, Irvine, California) or the Merci device (Concentric Medical, Mountain View, California). SWIFT had a roll-in phase during which all patients were treated with a stent retriever, and these patients were included in the current study. STAR was a prospective, multicenter, single-arm study from 2010 to 2012 in which patients with an occlusion of the anterior circulation (intracranial and terminus internal carotid artery and M1 and M2 segments of the middle cerebral artery) were included. All patients in STAR were treated with a stent retriever (Solitaire). In both SWIFT and STAR, patients were eligible only if MT was possible within 8 hours of symptom onset. In SWIFT PRIME, patients with an acute ischemic stroke and confirmed occlusion of the anterior intracranial circulation were randomized between intravenous tPA followed by MT with a stent retriever or intravenous tPA alone. The trial ran from December 2012 until Novem- ber 2014. In all 3 studies, clinical outcome was determined at 90-day follow-up by using the modified Rankin Scale.

We compared outcomes and technical details of patients with an M2 occlusion with those with an M1 occlusion. We excluded patients from SWIFT treated with the Merci device and patients from the control arm of SWIFT PRIME. Patients with a combined M1 and M2 occlusion were categorized as having M1 occlusion. Those with an occlusion at another location (eg, carotid terminus) were excluded from the analysis. The local ethics committee at every site approved the study protocol, and all patients or their legal representatives gave written informed consent.

Outcome data were adjudicated by an independent CT and MR imaging core laboratory, an angiography core laboratory, and a clinical events committee. The angiography core laboratory assessed the location of the occlusion. Table 1 describes the definition for each segment of the middle cerebral artery used by the core laboratory of the 3 studies. Other variables scored by the core laboratory or Clinical Events Committee were final revascularization grades, hemorrhagic complications, and other adverse events. In SWIFT and SWIFT PRIME, the assessors were blinded to study group assignments.

\section{MT Procedure}

The MT procedure used in STAR, SWIFT, and SWIFT PRIME has been described previously. ${ }^{7-9}$ The aim was to achieve successful recanalization of the territory of the occluded vessel. The use of a balloon-guide catheter was mandatory in STAR and SWIFT and optional in SWIFT PRIME. Up to 3 passes with the stent retriever were allowed according to the protocols. The decision to perform any additional passes was at the discretion of the interventionalist and was scored as a protocol violation. The procedure could be performed with the patient under local or general anesthesia. Follow-up brain imaging was performed after 24 hours in all patients.

\section{Data Analysis}

We compared the technical details of the procedure, complication rate, and clinical outcome at follow-up between patients with an M1 versus M2 occlusion. Successful reperfusion was defined as a modified Thrombolysis in Cerebral Infarction score of $\geq 2 b$ of the target territory. Both the proportions of symptomatic and any intracranial hemorrhage are provided. Symptomatic intracranial hemorrhage was defined as hemorrhage within 24 hours associated with an increase on the NIHSS of $\geq 4$ points or that resulted in death. Clinical outcome at 90 days is provided as mRS $0-1$, mRS 0-2, and all-cause mortality.

All analyses were by intention-to-treat. Categoric variables 


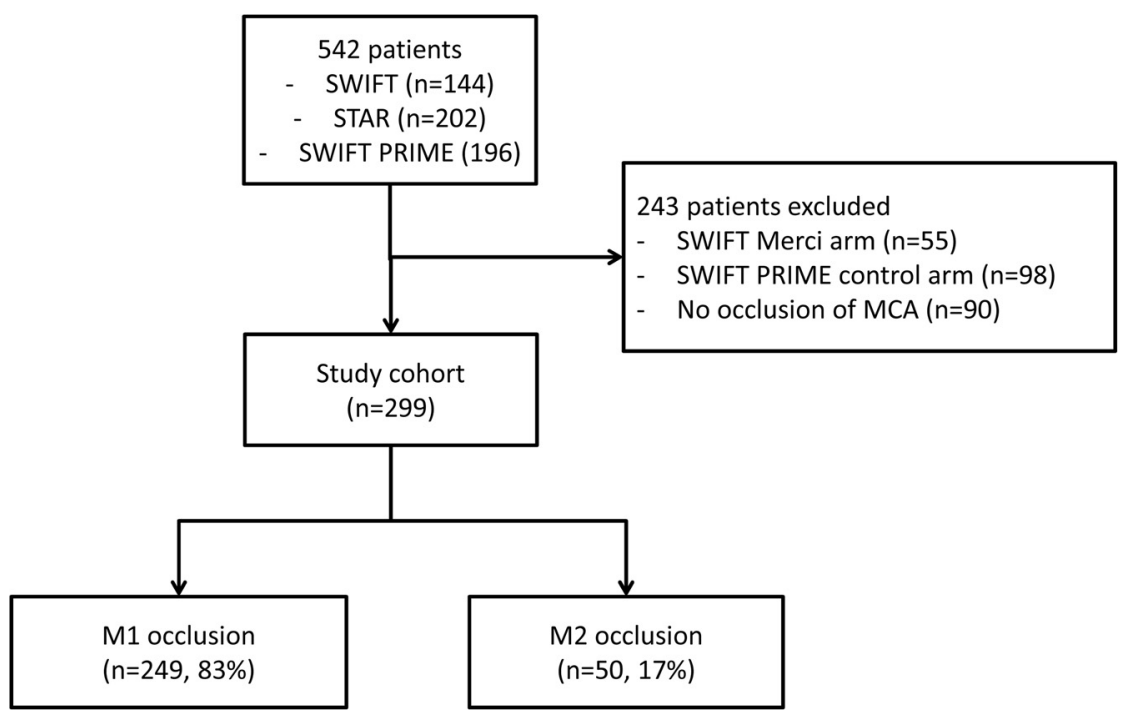

FIG 1. Flowchart of patient selection.

Table 2: Baseline characteristics ${ }^{\mathrm{a}}$

\begin{tabular}{lccc} 
& $\begin{array}{c}\text { M2 Occlusion } \\
(\boldsymbol{n}=\mathbf{5 0})\end{array}$ & $\begin{array}{c}\text { M1 Occlusion } \\
(\boldsymbol{n}=\mathbf{2 4 9})\end{array}$ & $\boldsymbol{P}$ Value \\
\hline Age (yr) (mean) & $71 \pm 11$ & $67 \pm 13$ & .04 \\
Female & $48 \%(24 / 50)$ & $57 \%(143 / 249)$ & .28 \\
NIHSS score (median) (IQR) & $13(10-17)$ & $17(14-20)$ & $<.001$ \\
Medical history & & & \\
$\quad$ Atrial fibrillation & $38 \%(19 / 50)$ & $37 \%(93 / 249)$ & 1.000 \\
Hypertension & $70 \%(35 / 50)$ & $61 \%(152 / 249)$ & .27 \\
Diabetes & $18 \%(9 / 50)$ & $15 \%(38 / 249)$ & .67 \\
Hyperlipidemia & $30 \%(15 / 50)$ & $37 \%(93 / 249)$ & .42 \\
Peripheral artery disease & $0 \%(0 / 50)$ & $4 \%(9 / 249)$ & .37 \\
Current smoker & $14 \%(7 / 49)$ & $21 \%(53 / 249)$ & .33 \\
Prior stroke/TIA & $12 \%(6 / 50)$ & $17 \%(42 / 249)$ & .53 \\
Systolic BP (mean) (mm Hg) & $150 \pm 28$ & $147 \pm 23$ & .45 \\
Diastolic BP (mean) (mm Hg) & $84 \pm 17$ & $80 \pm 15$ & .11 \\
Left-sided occlusion & $54 \%(27 / 50)$ & $47 \%(117 / 249)$ & .44 \\
Baseline ASPECTS (mean) & $9.0 \pm 1.3$ & $8.2 \pm 1.7$ & .003 \\
Receipt of IV tPA & $77 \%(36 / 47)$ & $69 \%(161 / 234)$ & .38 \\
Laboratory data & & & \\
Baseline serum glucose level (mean) & $127 \pm 51$ & $128 \pm 55$ & .89 \\
INR (mean) & $1.1 \pm 0.5$ & $1.1 \pm 0.3$ & .95 \\
Platelets (mean) & $235 \pm 75$ & $234 \pm 79$ & .95 \\
aPTT (mean) (sec) & $31 \pm 20$ & $29 \pm 22$ & .44 \\
\hline
\end{tabular}

Note:-IQR indicates interquartile range; BP, blood pressure; INR, international normalized ratio; aPTT, activated partial thromboplastin time.

${ }^{a}$ Categoric variables are given as percentages with $n / N$ in parentheses, where $n$ is the number of patients in whom the variable was present and $\mathrm{N}$ the total number of patients for whom that particular variable was reported.
The sponsor had no role in the preparation of the article. The corresponding author had full access to all the data in the study and had final responsibility for the decision to submit for publication. The studies were registered at ClinicalTrials.gov, numbers NCT01054560 (SWIFT), NCT01327989 (STAR), and NCT01657461 (SWIFT-PRIME).

\section{RESULTS}

Of the 542 patients enrolled in SWIFT, STAR, and SWIFT PRIME, 243 patients were excluded because they were allocated to the Merci arm of SWIFT ( $n=$ $55)$ or the control arm of SWIFT PRIME $(n=98)$ or because they did not have an occlusion of the middle cerebral artery ( $n=90$, Fig 1). Of the 299 included patients, 50 had an M2 occlusion; and 249, an M1 occlusion. Patients with an M2 occlusion were older (71 versus 67 years, $P=.04)$ and had a lower median NIHSS score (13 versus 17, $P<.001$ ) compared with patients with an M1 occlusion (Table 2). There were no important differences in medical histories between the groups. Baseline ASPECTS was higher in those with an M2 occlusion (9.0 versus $8.2, P=.003)$. The proportion of patients who received intravenous tPA before MT did not differ significantly (77\% versus $69 \%, P=.38$ ).

Time from groin puncture to recanalization was nonsignificantly shorter in patients with an M2 occlusion (median, 29 versus 35 minutes; $P=.41$, Table 3 ). The average number of passes with the stent retriever was also nonsignificantly lower in in patients with an M2 occlusion (mean, 1.4 versus $1.7 ; P=.07$ ). There was no difference in the proportion of patients in whom modified TICI $\geq 2 \mathrm{~b}$ was achieved ( $85 \%$ versus $82 \%$, were compared between groups by using a Fisher exact test. Continuous variables were compared by using a $t$ test, except in the cases in which medians and interquartile ranges are reported; then, a Wilcoxon rank sum test was used. Statistical analyses were conducted in SAS, Version 9.3 (SAS, Cary, North Carolina).

\section{Role of the Funding Source}

In all 3 studies, an academic steering committee supervised the trial design and operations. The sponsor of the study (Covidien) was responsible for site management, data management, and safety reporting. The study data were independently monitored. The statistical analyses were prepared by independent external statisticians (J. Schafer; Namsa, Minneapolis, Minnesota; S Brown, Altair Biostatistics, St. Louis Park, Minnesota).
$P=.82)$ or in the incidence of symptomatic intracranial hemorrhages (both 2\%). An mRS of $0-1$ at 90 days was achieved in 50\% of patients with an M2 occlusion, compared with $41 \%$ in those with an $\mathrm{M} 1$ occlusion $(P=.27)$.

\section{DISCUSSION}

We examined the feasibility and safety of MT with a stent retriever in patients with an isolated M2 occlusion. We found no significant differences in the reperfusion rate or risk of complications between patients with an M1 or M2 occlusion. If anything, there was a trend toward a lower number of passes required with stent retrievers in patients with an M2 occlusion. Clinical outcomes were nonsignificantly better in patients with an M2 occlusion, 
Table 3: Details of thrombectomy procedure and clinical and safety outcomes ${ }^{\mathrm{a}}$

\begin{tabular}{|c|c|c|c|}
\hline & $\begin{array}{l}\text { M2 Occlusion } \\
(N=50)\end{array}$ & $\begin{array}{l}\text { M1 Occlusion } \\
(N=249)\end{array}$ & $P$ Value \\
\hline $\begin{array}{l}\text { Time from groin puncture to recanalization } \\
\text { (min) (median) (IQR) }\end{array}$ & $29(22-45)$ & $35(25-52)$ & .41 \\
\hline No. of passes with stent retriever (mean) & $1.4 \pm 0.8$ & $1.7 \pm 1.0$ & .07 \\
\hline$\geq 3$ Passes with stent retriever & $13 \%(5 / 38)$ & $23 \%(52 / 227)$ & .21 \\
\hline $\mathrm{mTICl} 2 \mathrm{~b}$ or 3 reperfusion & $85 \%(34 / 40)$ & $82 \%(193 / 235)$ & .82 \\
\hline Rescue therapy & $6 \%(3 / 50)$ & $8 \%(19 / 249)$ & 1.000 \\
\hline \multicolumn{4}{|l|}{ Complications } \\
\hline Device-related serious adverse events & $6 \%(3 / 50)$ & $4 \%(10 / 249)$ & .46 \\
\hline Symptomatic ICH & $2 \%(1 / 50)$ & $2 \%(5 / 249)$ & 1.000 \\
\hline \multicolumn{4}{|l|}{ Outcome at 90-day follow-up } \\
\hline mRS 0-1 & $50 \%(25 / 50)$ & $41 \%(100 / 243)$ & .27 \\
\hline mRS 0-2 & $60 \%(30 / 50)$ & $56 \%(136 / 243)$ & .64 \\
\hline Mortality & $12 \%(6 / 50)$ & $10 \%(25 / 249)$ & .62 \\
\hline
\end{tabular}

Note:- mTICl indicates modified Thrombolysis in Cerebral Infarction; ICH, intracerebral hemorrhage; IQR, interquartile range.

${ }^{\text {a }}$ Categoric variables are given as percentages with $n / N$ in parentheses, where $n$ is the number of patients in whom the variable was present, and $N$ is the total number of patients for whom that particular variable was reported.

though this result most likely is because these patients had milder strokes, as shown by the lower baseline NIHSS scores.

Few studies have previously examined endovascular treatment of patients with an M2 occlusion, and in most of these, intraarterial thrombolysis or earlier generation thrombectomy devices were used. A post hoc analysis of the Prolyse in Acute Cerebral Thromboembolism (PROACT) II, Interventional Management of Stroke (IMS), and IMS II studies found successful reperfusion in approximately half of all patients with an M2 occlusion. ${ }^{10}$ Shi et $\mathrm{al}^{11}$ studied 28 patients with an M2 occlusion who were enrolled in the Mechanical Embolus Removal in Cerebral Ischemia (MERCI) and multi-MERCI trials. They found that compared with patients with an M1 occlusion, the number of required passes with the thrombectomy device was lower in those with an M2 occlusion, similar to our data. They also found higher recanalization rates in patients with an M2 occlusion. Sheth et $\mathrm{al}^{12}$ collected data on M2 occlusions from their single-center cohort on MT. They found no difference in recanalization rates between patients with an M2 or M1 occlusion, but the risk of intracranial hemorrhagic complications was lower in patients with an M2 occlusion. However, this observation could be related to different endovascular techniques that were used in each group. Most patients with an M2 occlusion were treated with intra-arterial tPA, while the Merci device was predominantly used in patients with an M1 occlusion. Various randomized trials have shown that the methods of endovascular reperfusion that were used in the abovementioned studies do not improve clinical outcome in patients. ${ }^{13-15}$ With the introduction of stent retrievers, these older techniques are now rarely applied; thus, the external validity of these studies is now limited.

Only 2 recent retrospective studies have examined the use of stent retrievers for patients with an M2 occlusion. Dorn et $\mathrm{al}^{16}$ reported outcomes of 15 patients with an M2 occlusion from a single-center retrospective registry. Similar to our results, their data do not suggest that patients with an M2 occlusion have a higher risk of procedural complications. The other study used data from a regional stroke registry. ${ }^{17}$ Most of the patients in this series were treated with a stent retriever, though other techniques were used in $14 \%$ of patients. Their rate of symptomatic intrace- rebral hemorrhage was $9 \%$, which is somewhat higher than that in our study. However, they did not assess patients with an M1 occlusion for comparison; this feature makes interpretation more difficult. Their patients were also in a more severe clinical condition, with a median NIHSS score of 16 , which is comparable with the NIHSS scores of the patients with an M1 occlusion in our study. A drawback of both studies is that they did not use an independent core laboratory to adjudicate the angiographic data and no information is provided on the definition that was used for an M2 occlusion.

Our study had no control group of medically treated patients available for comparison. As a result, we cannot determine whether MT with a stent retriever is superior to best medical management in patients with an M2 occlusion. Individually, neither of the recent thrombectomy trials has a sufficient number of patients with an M2 occlusion to answer this question. One issue that is important in any study that examines MT for M2 occlusion is a clear description of the definition of an M2 branch. The original classification by Fischer in $1938^{18}$ designated the M1 segment as terminating at the genu where the MCA takes a $90^{\circ}$ upward turn around the limen insulae; this definition was used in the STAR, SWIFT, and SWIFT PRIME studies. The M1 segment is then further divided into prebifurcation and postbifurcation segments with up to $82 \%$ of MCAs reported to bifurcate proximal to the genu. ${ }^{19,20}$ This terminology creates some degree of confusion because postbifurcation branches proximal to the genu are commonly designated as M2 segments.

There are also differences in the number and dominance of the divisions of the MCA, meaning that not all M2 divisions are the same with regard to the volume or importance of the territory that they supply. The variation in size of the divisions also means that technically not all M2 branches are accessible for mechanical thrombectomy. M2 divisions are smaller; therefore, navigating a microcatheter and deploying a stent retriever can be more challenging and associated with greater risk. Thus, when reviewing the patients included in this study, one must consider that the M2 occlusions were likely selected because they had features that made them favorable for mechanical thrombectomy, either because of their size or because they were critical to recanalize due to their supply to and area of eloquence. Patients also were required to have an NIHSS score of least an 8, which decreases the probability of smaller M2 branches being included. This selection of M2 occlusions is an important limitation of this study, and one that probably applies to all studies that examined MT for M2 occlusions.

Only high-volume stroke centers with extensive experience with MT participated in the SWIFT, STAR and SWIFT PRIME studies. In SWIFT, only centers that had participated in the MERCI or multi-MERCI trials or who had an annual MT volume of at least 30 patients were eligible. In STAR, the steering committee selected centers with 24-hour availability of MT that were 
experienced with the use of stent retrievers. As a result, caution is required when generalizing these results to stroke centers in general. On the other hand, given the positive results of the recent trials, MT volumes and thus experience of neurointerventionalists are likely to increase substantially in stroke centers within the next few years.

\section{CONCLUSIONS}

In this study, we examined the technical aspects, safety, and outcomes of mechanical thrombectomy in patients with an isolated M2 occlusion who were included in 3 large multicenter prospective studies. Our data suggest mechanical thrombectomy with a stent retriever can be considered in selected patients with acute ischemic stroke and an isolated M2 occlusion.

\section{ACKNOWLEDGMENTS}

The authors would like to thank Jill Schafer and Scott Brown for their help with the data analysis.

Disclosures: Jonathan M. Coutinho-UNRELATED: Grants/Grants Pending: Remmert Adriaan Laan Fonds, Comments: nonprofit organization for neurological research. David S. Liebeskind-RELATED: Grant: National Institutes of Health-National Institute of Neurological Disorders and Stroke, ${ }^{*}$ Comments: research grant; UNRELATED: Consultancy: Stryker,* Covidien,* Comments: core lab. Raul G. Nogueira-RELATED: Other: Covidien: SWIFT and SWIFT PRIME Trials Steering Committee (modest), STAR Trial core lab (significant); UNRELATED: Other: Stryker Neurovascular: Thrombectomy Revascularisation of Large Vessel Occlusions in Acute Ischaemic Stroke Trial (TREVO 2) Principal Investigator (modest) and Trevo and Medical Management Versus Medical Management Alone in Wake Up and Late Presenting Strokes (DAWN) Trial Principal Investigator (not compensated); Penumbra: 3D Separator Trial Executive Committee. ADDITIONAL: Dr Nogueira serves as a scientific consultant regarding trial design and conduct to Covidien, has served as the Principal Investigator for the TREVO 2 and DAWN trials, is part of the Penumbra 3D Separator Trial Executive Committee, has served as a data safety and monitoring board member for Stroke Trial with Rapid Medical, and is the editor-in-chief of the Interventional Neurology journal. Blaise W. Baxter-UNRELATED: Consultancy: Covidien, Penumbra, Stryker, Silk Road; Payment for Lectures (including service on Speakers Bureaus): Covidien, Penumbra, Stryker, Silk Road; Patents (planned, pending or issued): United States: 8,622,992 Devices and Methods for Perfusion Therapy; Travel/Accommodations/Meeting Expenses Unrelated to Activities Listed: Covidien, Penumbra, Stryker, Silk Road. Antoni Davalos_UNRELATED: Grants/Grants Pending: Covidien (unrestricted grant for REVASCAT trial)*; OTHER: Dr Davalos has served as a scientific consultant regarding trial design and conduct to Covidien. Alain Bonafé-UNRELATED: Consultancy: Covidien, ${ }^{\star}$ Comments: scientific consultant regarding trial design and conduct; Payment for Lectures (including service on Speakers Bureaus): Covidien. Reza Jahan-RELATED: Consulting Fee or Honorarium: Covidien Neurovascular; Support for Travel to Meetings for the Study or Other Purposes: Covidien Neurovascular; UNRELATED: Consultancy: Covidien; Payment for Lectures (including service on Speakers Bureaus): Covidien; OTHER: University of California Regents receive funding for Dr Jahan's services as a scientific consultant regarding the trial design and conduct to Covidien, and he is an employee of the University of California, which holds a patent on retriever devices for stroke. Jan Gralla—RELATED: Consulting Fee or Honorarium: Covidien, ${ }^{*}$ Comments: former Global Principal Investigator of the STAR study, consultant for Covidien. Jeffrey L. Saver-RELATED: Grant: Covidien*; Consulting Fee or Honorarium: Covidien*; Support for Travel to Meetings for the Study or Other Purposes: Covidien*; UNRELATED: Consultancy: Stryker,* Neuravia*; Patents (planned, pending or issued): University of California*; OTHER: University of California Regents receive funding for Dr Saver's services as a scientific consultant regarding the trial design and conduct to Covidien and Stryker, and he is an employee of the University of California, which holds a patent on retriever devices for stroke. Vitor Mendes PereiraUNRELATED: Consultancy: Covidien, ${ }^{*}$ Comments: as a Principal Investigator of the STAR trial; OTHER: Dr Pereira serves as a scientific consultant regarding trial design and conduct to Covidien and has served as the Global Principal Investigator for the STAR trial. Elad I. Levy_RELATED: Consulting Fee or Honorarium: Covidien, Comments: SWIFT Steering Committee member, SWIFT PRIME Executive Committee member as the US Interventional Principal Investigator; UNRELATED: Consultancy: Abbott (carotid training sessions); Expert Testimony: renders medical/legal opinion; Stock/Stock Options: Intratech Medical, Blockade Medical. Osama O. Zaidat-
UNRELATED: Consultancy: Covidien; Expert Testimony: Tanoury, Nauts, McKinney \& Garbarino, PLLC*; Grants/Grants Pending: Stryker, Hackney, Grover, Hoover \& Bean, PLC, ${ }^{*}$ Covidien, ${ }^{*}$ Genentech, ${ }^{*}$ MicroVention, ${ }^{*}$ Comments: research grants; OTHER: Dr Zaidat serves as a consultant to Covidien. Adnan H. Siddiqui-RELATED: Consulting Fee or Honorarium: Covidien, ${ }^{*}$ Comments: SWIFT PRIME Steering Committee member, Advisory Board; UNRELATED: Consultancy: Codman \& Shurtleff, Guidepoint Global Consulting, Penumbra (Penumbra 3D Separator Trial Steering Committee member; received honoraria), Stryker, Pulsar Vascular, MicroVention Flow-Redirection Endoluminal Device trial Steering Committee member, Lazarus Effect, Blockade Medical, Reverse Medical, W.L. Gore \& Associates; Payment for Lectures (including service on Speakers Bureaus): Codman \& Shurtleff; Other: Financial Interests: Lazarus Effect, Blockade Medical, Hotspur, Intratech Medical, StimSox, Valor Medical, Medina Medical; Advisory Board: Intersocietal Commission for the Accreditation of Vascular Laboratories, Medina Medical; Honoraria: Abbott Vascular, Toshiba American Medical Systems; OTHER: Dr Siddiqui serves as a consultant to Covidien. Mayank Goyal—RELATED: Consulting Fee or Honorarium: Covidien, Comments: SWIFT PRIME Executive Committee member as the Global Imaging and Workflow Principal Investigator; also received honoraria for speaking and teaching engagements; UNRELATED: Grants/Grants Pending: Covidien, ${ }^{*}$ Comments: Dr Goyal is one of the Principal Investigators for the ESCAPE Trial, which was partially funded by Covidien through an unrestricted grant to the University of Calgary; Patents (planned, pending or issued): GE Healthcare, Comments: systems and methods for diagnosing strokes, multiphase CTA technology: licensed for further development and marketing of technology; OTHER: Dr Goyal serves as a consultant to Covidien. *Money paid to the institution.

\section{REFERENCES}

1. Berkhemer OA, Fransen PS, Beumer D, et al. A randomized trial of intraarterial treatment for acute ischemic stroke. N EnglJ Med 2015; 372:11-20 CrossRef Medline

2. Goyal M, Demchuk AM, Menon BK, et al; ESCAPE Trial Investigators. Randomized assessment of rapid endovascular treatment of ischemic stroke. N Engl J Med 2015;372:1019-30 CrossRef Medline

3. Saver JL, Goyal M, Bonafe A, et al; SWIFT PRIME Investigators. Stent-retriever thrombectomy after intravenous t-PA vs. t-PA alone in stroke. N Engl J Med 2015;372:2285-95 CrossRef Medline

4. Jovin TG, Chamorro A, Cobo E, et al; REVASCAT Trial Investigators. Thrombectomy within $\mathbf{8}$ hours after symptom onset in ischemic stroke. N Engl J Med 2015;372:2296-306 CrossRef Medline

5. Campbell BC, Mitchell PJ, Kleinig TJ, et al; EXTEND-IA Investigators. Endovascular therapy for ischemic stroke with perfusion-imaging selection. N Engl J Med 2015;372:1009-18 CrossRef Medline

6. del Zoppo GJ, Poeck K, Pessin MS, et al. Recombinant tissue plasminogen activator in acute thrombotic and embolic stroke. Ann Neurol 1992;32:78-86 CrossRef Medline

7. Saver JL, Jahan R, Levy EI, et al. Solitaire flow restoration device versus the Merci retriever in patients with acute ischaemic stroke (SWIFT): a randomised, parallel-group, non-inferiority trial. Lancet 2012;380:1241-49 CrossRef Medline

8. Pereira VM, Gralla J, Davalos A, et al. Prospective, multicenter, single-arm study of mechanical thrombectomy using Solitaire flow restoration in acute ischemic stroke. Stroke 2013;44:2802-07 CrossRef Medline

9. Saver JL, Goyal M, Bonafe A, et al; SWIFT PRIME Investigators. Solitaire $^{\mathrm{TM}}$ with the Intention for Thrombectomy as Primary Endovascular Treatment for Acute Ischemic Stroke (SWIFT PRIME) trial: protocol for a randomized, controlled, multicenter study comparing the Solitaire revascularization device with IV tPA with IV tPA alone in acute ischemic stroke. Int J Stroke 2015;10:439-48 CrossRef Medline

10. Rahme R, Yeatts SD, Abruzzo TA, et al. Early reperfusion and clinical outcomes in patients with M2 occlusion: pooled analysis of the PROACT II, IMS, and IMS II studies. J Neurosurg 2014;121:1354-58 CrossRef Medline

11. Shi ZS, Loh Y, Walker G, et al; MERCI and Multi-MERCI Investigators. Clinical outcomes in middle cerebral artery trunk occlusions versus secondary division occlusions after mechanical thrombectomy: pooled analysis of the Mechanical Embolus Removal in Cerebral Ischemia (MERCI) and Multi MERCI trials. Stroke 2010;41:953-60 CrossRef Medline 
12. Sheth SA, Yoo B, Saver JL, et al; UCLA Comprehensive Stroke Center. M2 occlusions as targets for endovascular therapy: comprehensive analysis of diffusion/perfusion MRI, angiography, and clinical outcomes. J Neurointerv Surg 2015;7:478-83 CrossRef Medline

13. Broderick JP, Palesch YY, Demchuk AM, et al; Interventional Management of Stroke (IMS) III Investigators. Endovascular therapy after intravenous t-PA versus t-PA alone for stroke. $N$ Engl J Med 2013;368:893-903 CrossRef Medline

14. Kidwell CS, Jahan R, Gornbein J, et al; MR RESCUE Investigators. A trial of imaging selection and endovascular treatment for ischemic stroke. N Engl J Med 2013;368:914-23 CrossRef Medline

15. Ciccone A, Valvassori L, Nichelatti M, et al; SYNTHESIS Expansion Investigators. Endovascular treatment for acute ischemic stroke. N Engl J Med 2013;368:904-13 CrossRef Medline

16. Dorn F, Lockau H, Stetefeld H, et al. Mechanical thrombectomy of
M2-occlusion. J Stroke Cerebrovasc Dis 2015;24:1465-70 CrossRef Medline

17. Flores A, Tomasello A, Cardona P, et al; Catalan Stroke Code and Reperfusion Consortium Cat-SCR. Endovascular treatment for M2 occlusions in the era of stentrievers: a descriptive multicenter experience. J Neurointerv Surg 2015;7:234-37 CrossRef Medline

18. Fischer E. Die lageabweichungen der vorderen hirnarterie im gefä $\boldsymbol{\beta b i l d . ~ Z e n t r a l b l ~ N e u r o c h i r ~ 1 9 3 8 ; 3 : 3 0 0 - 1 3 ~}$

19. Tanriover N, Kawashima M, Rhoton AL Jr, et al. Microsurgical anatomy of the early branches of the middle cerebral artery: morphometric analysis and classification with angiographic correlation. J Neurointerv Surg 2003;98:1277-90 Medline

20. Gibo H, Carver CC, Rhoton AL Jr, et al. Microsurgical anatomy of the middle cerebral artery. J Neurointerv Surg 1981;54:151-69 Medline 